\title{
Uso de energía alternativa en los desarrollos turísticos del Caribe
}

DOI: 10.22403/UQROOMX/TYP03/14

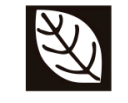

Miguel Breceda*/Eduardo Rincón*

Luis Carlos Santander**

\section{Resumen}

Frente a la disyuntiva entre el uso de energía de manera sustentable o convencional, se reseñan relevantes esquemas, mecanismos y proyectos nacionales e internacionales que canalizan recursos financieros en apoyo al desarrollo de la energía renovable. En materia turística en general y para el Caribe en particular, se identifican acciones concretas para la promoción de fuentes alternativas de energía. Finalmente, se argumenta la relevancia y viabilidad que para el desarrollo turístico del Caribe tienen los sistemas fotovoltaicos, fototérmicos, aerogeneradores y de biogás.

Palabras Energía alternativa, fuentes de energía, turismo sustentable, CLAVE $\mid$ tecnologías.

*Universidad Autónoma de la Ciudad de México

* Universidad de Quintana Roo / Isant@uqroo.mx 


\section{Energía y medio ambiente}

El consumo mundial neto de energía pasará de 13.3 billones de kilowatt hora en $200 \mathrm{I}$ a 23 billones en el 2025. Este incremento de $73 \%$ en el consumo de energía contrasta con una predicción de crecimiento de la economía mundial de $54 \%$ en el mismo periodo. También se prevé que el consumo de petróleo aumente de 77 millones de barriles al día a 121 millones; el consumo de gas natural en $67 \%$ y que el carbón continúe dominando la generación de electricidad en Asia y en particular en China. Con ello las emisiones de dióxido de carbono se incrementarán en $65 \%$, pasando de 24000 millones de toneladas métricas en 200 I a 37. I mil millones en 2025 (ENS, 2004). Las emisiones de gases invernadero son precisamente las responsables del calentamiento global, de las modificaciones del $\mathrm{pH}$ en los océanos y mares del mundo y de otras graves consecuencias. En el campo de la energía tenemos uno de los más penosos ejemplos de los patrones de consumo no sustentable que caracterizan a la sociedad contemporánea.

Frente al escenario de la insustentabilidad del modelo de desarrollo, hay propuestas y respuestas orientadas a la armonía entre desarrollo y medioambiente. Es importante establecer que no solamente se trata de propuestas de conservacionistas idealistas opuestos al desarrollo. Por ejemplo, la comisionada del Medio Ambiente de la Unión Europea ha señalado:"Cuando hablamos de 'productos verdes' y de 'innovación tecnológica' estamos hablando de 'negocios'.' (Wallström, 200 I). Con la evolución del paradigma del desarrollo sustentable, la cuestión ambiental ha dado un giro de 180 grados. La protección del medioambiente ya no es vista como una limitante al crecimiento y las inversiones, por el contrario, con las condiciones necesarias es una fuente de crecimiento y rentabilidad que orienta la cíclica reestructuración económica del sistema capitalista. Evidentemente, se trata de un proceso contradictorio donde el modelo de vanguardia atractivo para empresas líderes y globales desplaza a empresas e industrias obsoletas o con fuertes intereses, como puede ser el caso de los sectores petroleros de gas natural y carbón.

En este proceso de claras tendencias antagónicas, los organismos y agencias nacionales y multilaterales juegan un papel importante en el apoyo financiero, de gestión y promoción del modelo en pro de una presunta sustentabilidad energética. Por ejemplo, en el marco del Mecanismo de Desarrollo Limpio establecido en el protocolo de Kyoto, el Banco Mundial es la agencia líder en 
la promoción del mercado de carbono, donde con recursos de gobiernos y empresas de países desarrollados se financian proyectos de energía renovable o de reducción de los niveles de dióxido de carbono (como plantaciones de eucaliptos que lo absorban) en países en vías de desarrollo. Con estos esquemas, los países industrializados compran unidades de reducción de emisiones que se contabilizan a favor de sus obligaciones de reducir las emisiones de gases de efecto invernadero sin necesidad de disminuir realmente las emisiones de su planta productiva. Uno de varios esquemas específicos es el Fondo del Carbono para el Desarrollo Comunitario con más de mil millones de dólares para proyectos en Uganda; en Nepal, donde antes del 2009 se instalarán 162000 centrales de biogás; y en Honduras, donde se suministrará electricidad por primera vez a los 40000 habitantes de La Esperanza (Banco Mundial, 2005).

\section{Turismo sustentable $y$ energía alternativa}

El Plan para la Implementación de la Agenda 2 I define el turismo sustentable, en el que incluye al ecoturismo, como aquel que incrementa los beneficios para la población y la comunidad anfitriona, manteniendo la integridad cultural y ambiental, al tiempo que mejora la protección de las áreas ecológicamente sensibles y contribuye a fortalecer las comunidades rurales y locales.

En el terreno del turismo el paradigma de la sustentabilidad es un gran paraguas para mecanismos e instrumentos de financiamiento y promoción de proyectos de energía renovable. En este caso, una de las agencias más activas es el Programa de las Naciones Unidas para el Medio Ambiente (PNUMA), que entre muchos otros proyectos para desarrollos turísticos está financiando el de Turismo sustentable en el Sendero de Chile: una alternativa para la protección de los recursos naturales, que incorpora energía renovable para la iluminación, calentamiento, bombeo de agua y preparación de alimentos en las comunidades aledañas al sendero de $8000 \mathrm{~km}$ de largo (Conama s/f). Igualmente, el PNUMA, en colaboración con la Organización de Cooperación Económica de Asia Central, promueve y desarrolla fuentes de energía renovable en proyectos de turismo sustentable o ecoturismo (Europa Press, 2004).

Otro ejemplo de mecanismos promotores y facilitadores de la energía renovable en el turismo es la organización Energía Renovable y Desarrollo Sostenible (REST por sus siglas en inglés) conformado por I 2 agencias de países 
de la Unión Europea y cuyo objetivo final es alcanzar la eliminación total de emisiones de carbono por parte de hoteles interesados en atraer clientela con conciencia y compromiso ambiental. Un proyecto específico es el de la Agencia Florentina para la Energía, donde 58 hoteles del segmento del turismo rural instalarán paneles solares y sistemas de biomasa (REST s/f). Otro tipo de eco-etiqueta en materia de energía para hoteles es el llamado Sistema de Tu-rismo Responsable u Hoteles de la Biosfera que en asociación con la UNESco certifica establecimientos cuyo consumo de energía convencional se ha reducido sustancialmente con el uso de fuentes solar y eólica y con diseños arquitectónicos que obtienen las máximas ventajas de condiciones climáticas favorables para el uso térmico de los edificios.

En el caso específico del Caribe, donde la mayoría de los Estados son pequeñas islas, una referencia o marco importante para el desarrollo de proyectos turísticos con energía renovable es el Programa de Acción de Barbados. El Programa fue adoptado en 1994, y proporciona el marco internacional para promover el desarrollo sustentable con acciones concretas para auxiliar al desarrollo de los Estados insulares en áreas tales como energía. Con miras a canalizar recursos financieros para proyectos que permitan avances en el Programa de Acción de Barbados, el PNuma y la Comunidad del Caribe (Caricom) crearon en 2003 el Programa de los Pequeños Estados Insulares del Caribe que contempla un Fondo Regional de Sostenibilidad (PNUMA, 2004).

En el Caribe, el petróleo importado es la fuente primaria de la energía comercializada y es fundamental para la generación de electricidad y para el transporte. En varios de los Estados insulares el costo de la electricidad es relativamente alto, lo que frena la inversión. Una reducción en el costo de las importaciones de petróleo, debido a la utilización de energía renovable y a una mayor eficiencia energética, puede contribuir de forma relevante al mejoramiento social y ambiental.

Prácticamente en todo el Caribe hay importantes fuentes de energía renovable (solar, hidráulica, eólica, geotérmica, biomasa o biogás). Sin embargo, la conciencia sobre el potencial y eficiencia de la energía renovable es limitada en los sectores gubernamentales y empresariales. Sin duda, el turismo, por ser la industria más grande del mundo en términos económicos y de expansión, es un sector de importancia estratégica para el sector energético.

La cantidad de energía consumida no es el único elemento relevante, destacan el factor de estacionalidad, que implica capacidades de abastecimiento 
suficiente para los limitados períodos de ocupación a 100\% y el creciente "aislamiento" geográfico que caracteriza a nuevos nichos del mercado, como es el caso del ecoturismo. Los costos promedio en energía de un hotel, fluctúan entre 3 y $10 \%$ de los gastos totales y consumen la mayor cantidad de recursos después de los gastos de personal y alimentos. Por otra parte, Se calcula que en la construcción o renovación de un hotel sólo entre 8 y $10 \%$ del total de la inversión es requerido para calificarlo como sustentable en el campo energético (Islandonline, s/f).

La introducción de fuentes de energía renovable se debe complementar con diseños arquitectónicos ambientalmente eficientes. Sin embargo, el desconocimiento de las posibilidades en el campo de la energía renovable se traduce en falta de cooperación entre arquitectos, ingenieros, consultores, promotores, agentes financieros y gerentes de hoteles. Sin embargo, no debemos olvidar que el nicho de más alto crecimiento y con un perfil de turista con ingresos por arriba del promedio del de los viajantes internacionales es aquel que brinda entornos y comportamientos ambientalmente amigables.

\section{Sistemas económicos, confiables y eficientes para aprovechar las fuentes renovables de energía}

Desde el punto de vista energético, todo desarrollo sustentable está necesariamente basado en las fuentes renovables de energía, como la radiación solar y sus manifestaciones secundarias: el viento, la biomasa, la lluvia, etcétera.

Existen en el mercado sistemas altamente confiables, eficaces y económicos para aprovechar las fuentes renovables de energía, que son abundantes en el Caribe. En seguida se describen los que podrían emplearse en desarrollos turísticos, destacando sus ventajas sobre los sistemas convencionales.

Sistemas fotovoltaicos. Tan sólo en México se han instalados más de 60000 pequeños sistemas fotovoltaicos para generar electricidad en comunidades aisladas. Por lo general son sistemas de baja potencia, de alrededor de doscientos vatios cada uno. Sin embargo, existen ya instalaciones de megavatios y plantas comerciales para servicio público eléctrico con base en módulos fotovoltaicos. Los sistemas fotovoltaicos que generan electricidad convirtiendo directamente la 
luz solar en electricidad están basados en una tecnología que es completamente madura y está demostrado que es sumamente confiable, requiere de muy poco mantenimiento, no lleva partes móviles y tiene una vida útil garantizada superior a veinte años, por lo que a pesar de su relativo alto costo, es sumamente rentable, aun en localidades urbanas (en Estados Unidos, decenas de empresas grandes están aplicando masivamente los módulos fotovoltaicos aun cuando cuentan con el suministro eléctrico convencional).

Con mayor razón, en los desarrollos turísticos del Caribe deberían emplearse los sistemas fotovoltaicos, al menos para la iluminación nocturna. Además de producir un ahorro significativo de energía eléctrica de la red, asegura la iluminación durante los apagones que se producen cuando la red tiene algún problema. Más aún, la región del Caribe está expuesta a varios huracanes anuales, en los que la red casi con seguridad deja de funcionar. Se tiene la experiencia en la Florida de que la única fuente de energía eléctrica disponible después de algunos huracanes particularmente destructivos, han sido las luminarias de módulos fotovoltaicos.

Por supuesto que estos módulos pueden tener una aplicación mucho mayor que la mera iluminación nocturna, de hecho, podría electrificarse completamente un hotel con estos sistemas, lo que se justificaría en el caso de estar lejos de la red, en una pequeña isla, por ejemplo. Sin embargo, la inversión podría ser demasiado alta para hoteles y desarrollos que ya cuentan con electricidad convencional. En la figura I se muestra parte de la techumbre que tiene una hectárea de celdas fotovoltaicas con una capacidad de I.3 MW, en un edificio energéticamente sustentable, construido en madera y en cuyo interior se cuenta con áreas recreativas, palmeras, hoteles, bancos, escuela y otros servicios. La electricidad producida es diez veces más de la que requiere el edificio, por lo que los excedentes se venden a la compañía eléctrica. Este edificio, construido en Alemania -donde el recurso solar por metro cuadrado es menos de la mitad del disponible en el Caribe- es un ejemplo de que aun edificaciones muy grandes, que demandan enormes cantidades de electricidad, pueden ser enteramente electrificadas con energía fotovoltaica. En el caso de los desarrollos turísticos del Caribe, con sistemas mucho más pequeños y económicos, debería emplearse la electricidad de origen fotovoltaico que, como ya se mencionó, es sumamente confiable, insensible a los apagones, de gran calidad en cuanto a estar libre de armónicas y variaciones en el voltaje, su 


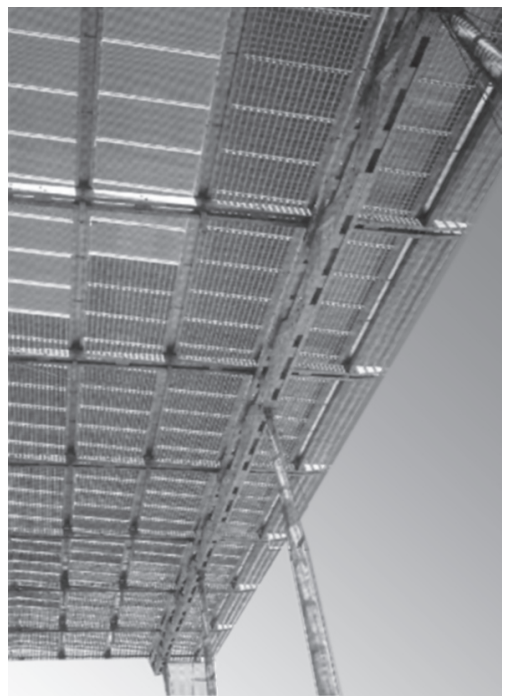

Figura I. Parte de una techumbre fotovoltaica de 10 mil metros cuadrados en Herne, Alemania

generación es completamente silenciosa, no requiere de combustibles fósiles, no emite gases tóxicos ni de invernadero y aprovecha un recurso gratuito y sumamente abundante en todo el Caribe: la energía solar.

Sistemas fototérmicos. La luz solar puede ser aprovechada de un modo mucho más económico que para generar electricidad: para el calentamiento de agua y otros fluidos para diversas aplicaciones; la más rentable ha demostrado ser la calefacción de las piscinas. Esta es una aplicación tan rentable que la inversión requerida para instalar los calentadores solares y demás aditamentos se recupera en los primeros dos años de servicio,y la vida útil de estos sistemas, por lo general, es superior a 14 años. Es sumamente recomendable el uso de calentadores solares en lugar de las calderas convencionales para calentar el agua de las piscinas de los desarrollos turísticos del Caribe.Asimismo, el agua caliente para las regaderas, tinas y jacuzis también debe provenir de calentadores solares. Éstos tienen la gran ventaja de no requerir de combustibles fósiles -que son caros, contaminantes, peligrosos y no siempre están disponibles-y de aprovechar la abundante energía solar durante todo el año. 
En la figura 2 se muestran diversos sistemas para el calentamiento de agua con colectores solares que pueden proveer agua caliente a $80^{\circ} \mathrm{C}$. Para albercas se requieren temperaturas mucho más bajas y los colectores pueden ser de materiales plásticos sin cubiertas, por lo que resultan sumamente baratos $y$ eficaces. Las albercas olímpicas de Atlanta, Sydney y Atenas fueron calentadas, con mucho éxito, con colectores solares de plástico.

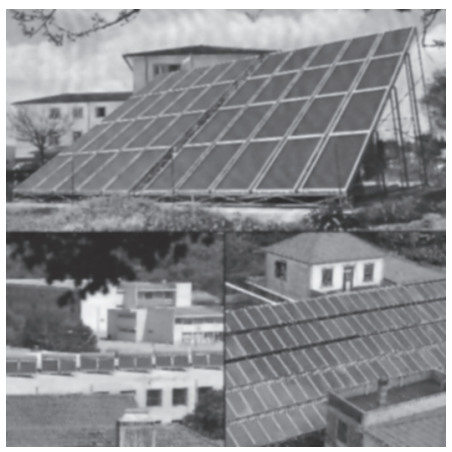

Figura 2. Diversas InSTALACIONES DE CALENTADORES SOLARES DE AGUA PARA USO RESIDENCIAL Y DE SERVICIOS

Otras aplicaciones poco conocidas del aprovechamiento de la energía solar como fuente de cocción son los hornos y las planchas solares, con los que es posible cocinar cualquier alimento sin necesidad de quemar combustibles fósiles ni leña. Esto tiene un gran atractivo ambiental y económico, ya que la cocina por lo general contribuye con más de $20 \%$ del consumo energético térmico en un desarrollo turístico. En las siguientes figuras se muestran un horno solar y un comal solar Tolokatsin -los más eficientes a nivel mundial- que han sido empleados en comidas solares preparadas para centenares de comensales. Los desarrollos turísticos del futuro deberían emplear en gran escala estos hornos y comales solares en beneficio del ambiente y de los usuarios, que se ahorrarían el combustible fósil o la leña, se evitarían el riesgo a sufrir quemaduras o intoxicaciones por respirar gases producto de la combustión o combustible no quemado y comerían más saludable y sabroso.

Pequeños aerogeneradores. Existen en el mercado pequeños aerogeneradores de menos de 600 vatios de potencia que pueden instalarse fácilmente para producir electricidad a partir del viento, recurso también muy abundante 


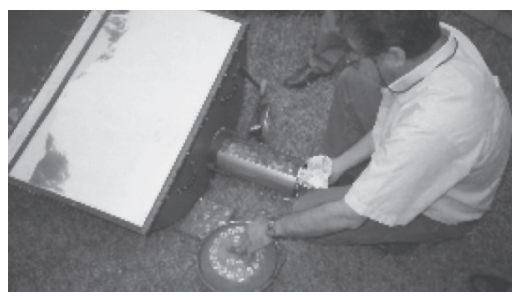

Figura 3. Horno solar Tolokatsin de 12 Litros de CAPACIDAD

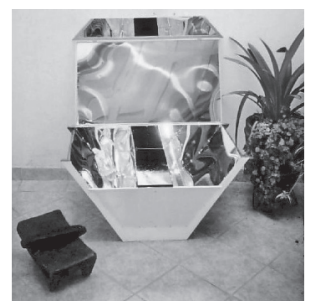

Figura 4. El comal solar Tolokatsin 2, empleado para freir alimentos

en el Caribe, principalmente en las zonas costeras. Estos sistemas tienen la gran ventaja de poder producir electricidad durante la noche, siempre que haya viento, por supuesto, y la electricidad producida es más barata que la generada con módulos fotovoltaicos. Su desventaja radica en que no siempre se tiene la seguridad de que se tendrán las velocidades del viento requeridas para la generación eléctrica. Lo más recomendable es emplearlos en conjunto con módulos fotovoltaicos de manera híbrida. Con esto se logra un efecto sinérgico: el beneficio del sistema aerogenerador más sistema fotovoltaico es superior al de la suma de los beneficios por separado. Esto por lo general es cierto cuando se emplean sistemas que aprovechan las fuentes renovables de energía, por lo que la recomendación sería emplear varias fuentes simultáneas de manera armónica para tener los mejores beneficios al menor costo.

Sistemas generadores de biogás. Los llamados biodigestores son sistemas muy confiables y sencillos que han sido empleados por millares en China, India y aun en los países europeos más desarrollados. Como su nombre lo sugiere, 
producen el llamado biogás -que químicamente es muy similar al gas natural-a partir de desechos orgánicos. Con esto se logran tres beneficios importantes: la producción de un combustible que puede ser empleado para calentamiento o generación de electricidad, la eliminación de desechos que pueden constituirse en focos insalubres y la obtención de un mejorador de suelos, que es en lo que se transforma la materia orgánica después de la producción de biogás, que puede ser usado en jardines y campos de cultivo.

En resumen, las fuentes renovables de energía tienen un inmenso campo de aplicación en los desarrollos turísticos de todo el mundo. En particular, el Caribe está magníficamente dotado de energía solar, energía eólica, biomasa y, aunque no tratado aquí, de energía oceánica, con las que es recomendable energizar los desarrollos turísticos para cuidar el ambiente, aprovechando un recurso disponible en toda el área, ahorrando en electricidad convencional y evitando combustibles fósiles.

\section{FUENTES CONSULTADAS}

Banco Mundial (2005). “Fondo de Carbono para el Desarrollo Comunitario recibe inesperado impulso: socios públicos y privados invierten US \$ 128 millones", comunicado de prensa número 2005/362/ESSD. Disponible en http://web.worldbank.org/WBSITE/EXTERNAL/ NEWS. [30 de marzo de 2005]

Conama (s/f). "Turismo sustentable en el Sendero de Chile", disponible en el sitio Internet de la Comisión Nacional del Medio Ambiente de Chile www.conama.cl [30 de marzo de 2005].

eNs (2004). "Asian Growth Fuels 20 Year Climb in World Energy Use”, Environmental News Service, I5 de abril. Disponible en http://www.ensnewswire.com/ens/apr2004-04-I5-03.asp [20 de abril de 2004].

Europa Press (2004). "PNUMA y ECO llegan a un acuerdo para fomentar las energías renovables y el turismo sostenible en Asia Central”, 19 de agosto. Disponible en www.finanzas.com/id.7808094/noticias/noticia. htm [26 de marzo de 2005].

Islandonline. s/f. "Sustainable Tourism and Renewable Energy Sources". Disponible en www.islandoline.org/island20I0/PDF [26 de marzo de 2005]. 
REST (s/f). Renewable Energy and Sustainable Tourism. Disponible en www.resthotels.org/en/ [26 de marzo de 2005].

PNUMA (2004). Futura aplicación del Programa de Acción de Barbados: colaboración entre el PNUMA y Caricom. Programa de las Naciones Unidas para el Medio Ambiente. Disponible en www.pnuma.org [30 de marzo de 2005].

Wallström, Margot (200I)."From Lisbon to Gothenburg. The Business Agenda for Sustainable Development", ponencia presentada en el seminario Achieving Effective Sustainable Development:The European Policy Agenda during the Swedish Presidency, marzo, celebrado en Londres. Disponible en http://europa.eu.int/comm/environment/press/index. [30 de marzo de 2005]. 


\section{Universidad de Quintana Roo Unidad Académiea Cozuumel División de Desarrollo Sustentade}

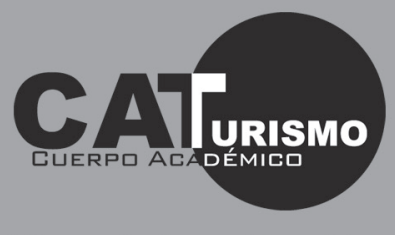

Línea de investigación: Turismo y Desarrollo

Cuerpo Académico
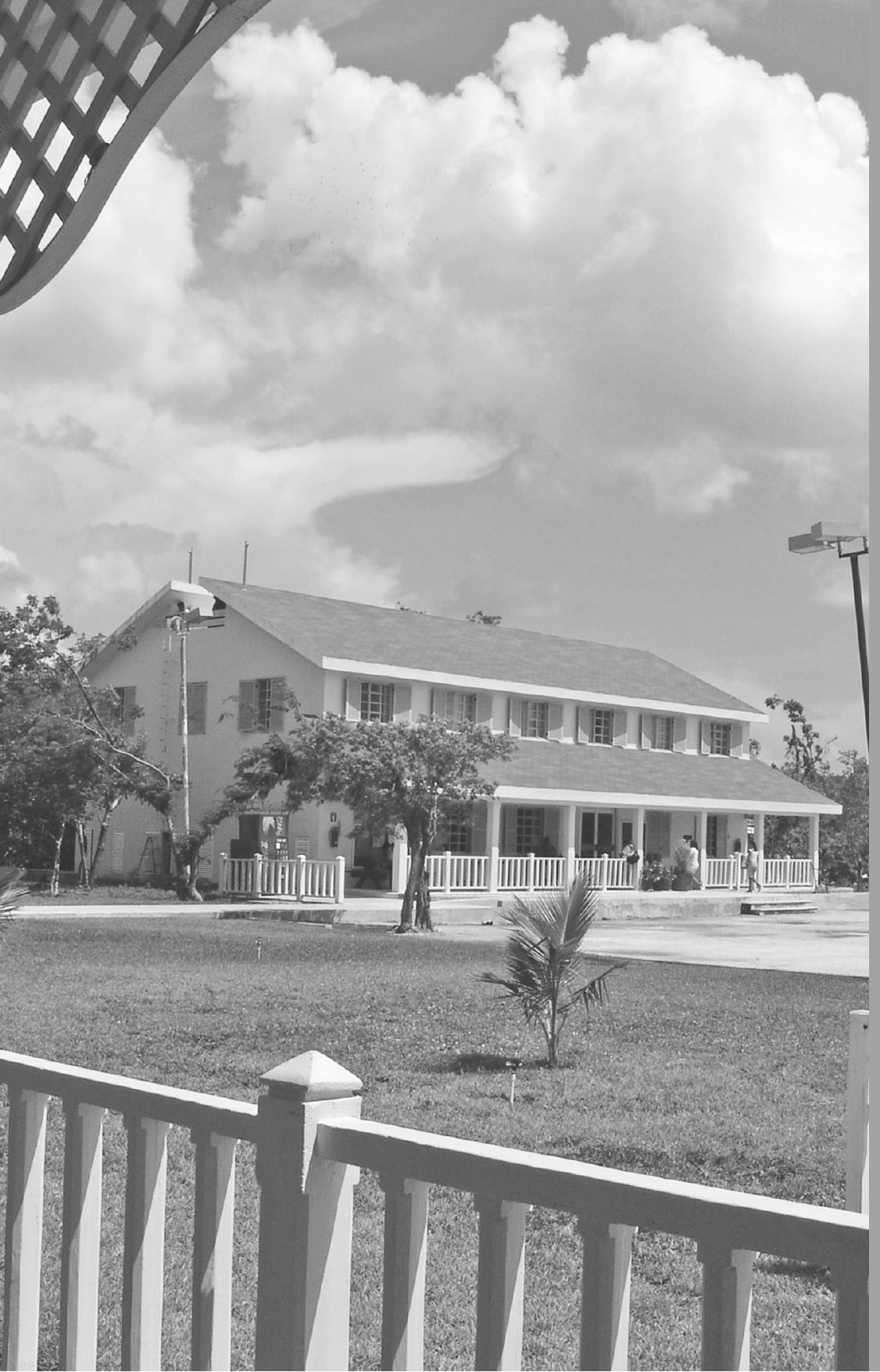

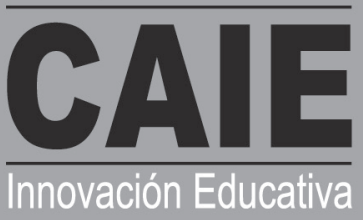

Línea de investigación: Educación

\section{CA marna}

Línea de investigación: Biología, manejo y conservación de los recursos naturales acuáticos 